\title{
On the Higher Thom Polynomials of Morin Singularities
}

\author{
Dedicated to Professor H. Toda on his 60th birthday
}

By

\section{Yoshifumi ANDO*}

\section{§o. Introduction}

In this paper we will give a formula to calculate generalized Thom polynomials defined by using Stiefel-Whitney or Chern homology classes of Morin singularities for mappings with only Morin ones.

Let $N$ and $P$ be smooth (resp. complex) manifolds of dimensions $n$ and $p$. Let $i=\max (1, n-p+1)$ and $I_{r}$ be the $r$ sequence $(i, 1, \ldots$, 1). A geometric interpretation of Morin singularities $S^{I}(f)$ of a smooth map $f: N \rightarrow P$ is as follows (cf. [9, 14]). Let $S^{i}(f)$ denote the set of all points $x$ of $N$ such that $\operatorname{rk}\left(\operatorname{Ker} d f_{x}\right)$ is $i$. If $S^{i}(f)$ is a submanifold of $N$, then we define $S^{i, j}(f)$ as the set $S^{j}\left(f \mid S^{i}(f)\right)$ similarly. We may continue to define $S^{I_{r}}(f)$ as $S^{1}\left(f \mid S^{I^{r-1}}(f)\right)$ inductively. A point of $S^{i, 0}(f)$ or $S^{I r}(f)(r \geqq 2)$ is called a Morin singularity of symbol $(i, 0)$ or $I_{r}$ respectively. By the elaborate approach due to Boardman [2] $S^{I_{r}}(f)$ becomes a submanifold for generic smooth maps in the real case. Let $f$ have only Morin singularities. Let $d$ be the codimension of $S^{I_{r}}(f)$ in $N$ and $s_{n-d-k}$, the $(n-d-k)$-th Stiefel-Whitney (resp. Chern) homology class of $S^{I_{r}}(f)$ 。 Let $i: S^{I_{r}}(f) \rightarrow N$ be the inclusion. Then it will be natural to consider the Poincaré dual of $i_{*}\left(s_{n-d-k}\right)$ in $H^{*}(N)$ and call it the $k$-th Thom polynomial of the Morin singularity of symbol $I_{r}$ for $f$. We denote it by $P\left(I_{r}, k, f\right)$. The 0 -th Thom polynomial is, of course, the well known Thom polynomial (see, [7, 14]).

As applications of the formula of Theorem 3.2 we will calculate

Communicated by N. Shimada, July 23, 1986.

*Department of Mathematics, Yamaguchi University, Yamaguchi 753, Japan. 
$P\left(I_{r}, k, f\right)$ for the case $n=p$ or $p+1$ and show several explicite formulas in Section 1. Although the notion of higher Thom polynomials is definable for general maps, its calculation seems extremely difficult. The known results concerning the calculation of Thom polynomials of higher order Thom-Boardman singularities of general maps are not much such as the explicite polynomial for $S^{i}(f)$ due to Porteous [10], the formula to calculate those of second order in a finite process (Ronga [11]) and others of some third order singularities (Damon [3]). In Gaffney [6] the Thom polynomial of $\overline{\overline{1}^{1111}}$ has been calculated. We seems far from the complete calculation even for the case $\overline{\Sigma^{1 \cdots 1}}$, although I. R. Porteous [10] has already shown the recurrence relation calculating $P\left(I_{r}, 0, f\right)$ for $n=p$ which looks different from ours on the surface because of the Thom polynomial of $\overline{\Sigma^{2}}$ (see also Sergeraert [12]). This is the reason why we need the above assumption on $f$. It seems very restrictive in general. However in the problem of putting an immersion of an $n$ manifold into an $n+1$ manifold with tangent line subbundle $X$ in a general position with respect to $X$, we only need to consider Morin singularities. The result of this paper will be applied to this problem in the forthcoming paper. It has also been shown in [1] that $P\left(I_{r}, 0, f\right)$ is an important invariant in eliminating Morin singularities of symbol $I_{r}$ from $f$. We display three Thom polynomials of $S^{I_{4}}(f)$ :
a) $6 \bar{c}_{4}+9 \bar{c}_{3} \bar{c}_{1}+2 \bar{c}_{2}^{2}+6 \bar{c}_{2} \bar{c}_{1}^{2}+\bar{c}_{1}^{4}$ (Gaffney [6, Theorem 2.2])
b) $P_{1}^{4}(f)=6 \bar{c}_{4}+8 \bar{c}_{3} \bar{c}_{1}+3 \bar{c}_{2}^{2}+6 \bar{c}_{2} \bar{c}_{1}^{2}+\bar{c}_{1}^{4}$ (Porteous [10])
c) $P\left(I_{4}, 0, f\right)=6 \bar{c}_{4}+19 \bar{c}_{3} \bar{c}_{1}-8 \bar{c}_{2}^{2}+6 \bar{c}_{2} \bar{c}_{1}^{2}+\bar{c}_{1}^{4}$ (see (1.2))

(a) is valid for maps having other singularities than Morin ones. They are different by the Thom polynomial, $\bar{c}_{2}^{2}-\bar{c}_{3} \bar{c}_{1}$ of $\overline{\Sigma^{2}}$.

In Section 2 we will review the definition of so called Boardman's manifold in the infinite jet space $J(N, P)$. In Section 3 we will lift $S^{I_{r}}(f)$ up to a submanifold $\tilde{S}^{I_{r}}(f)$ of a certain flag bundle whose normal bundle is extended to one over the whole space. The formula of Theorem 3. 2 follows from projecting the dual class of $s_{n-d-k}\left(\tilde{S}^{I_{r}}(f)\right)$ by the Gysin homomorphism. This idea comes from the method for $r=1$ or 2 in [10] and [11].

The author has been indebted to Fukuda [5] in defining the 
higher Thom polynomials and would like to thank Professor T. Gaffney who answered to his question on the disagreement of the formula of [6, Theorem 3.2] and that of [12].

\section{$\S 1$. Formulas}

By using the formula of Theorem 3.2 together with Proposition 4.1 and the result of [4] we can calculate $P\left(I_{r}, k, f\right)$ in terms of characteristic classes of $N$ and $P$ in a finite process. We will prove the following in Section 4. Let $c=1+c_{1}+c_{2}+\ldots$ be the StiefelWhitney (resp. Chern) class and $\bar{c}=1+\bar{c}_{1}+\bar{c}_{2}+\ldots$, its formal inverse.

Let $n=p$. We formally write as $c(N)=\prod_{i=1}^{n}\left(1+a_{i}\right)$ and $f^{*}(c(P))=$ $\prod_{i=1}^{n}\left(1+b_{i}\right)$ by variables $a_{i}$ and $b_{i}$ with degree 1 (resp. 2). Let $x$ and $y$ be of degree 1 (resp. 2). Let $f_{1}$ be the following polynomial;

$$
\prod_{i=1}^{n}\left(b_{i}-x\right)\left\{\sum_{l=0}^{n-1}\left(\sum_{j=0}^{n-l-1}(-1)^{j} c_{j}(N) x^{n-l-j-1}\right) y^{l}\right\} \prod_{t=2}^{r}(-t x+y)
$$

and $f_{2}$, the sum of all monomials of degree $k$ (resp. $2 k$ ) of the following;

$$
\begin{aligned}
\left\{\prod_{i=1}^{n}\left(1+a_{i}\right)\right. & \left.\left(1+a_{i}-x\right)\left(1-b_{i}+y\right)\left(1+b_{i}-x\right)^{-1}\left(1-a_{i}+y\right)^{-1}\right\} \\
& \times(1-x+y) \prod_{t=2}^{r}(1-t x+y)^{-1} .
\end{aligned}
$$

We write the product $f_{1} f_{2}$ as

$$
\sum_{s, t \geq 0} A_{s, t}\left(a_{i}, b_{i}\right) x^{s} y^{t} \text {. }
$$

Similarly let the product of two polynomials $\prod_{i=1}^{n}\left(b_{i}-x\right)$ and the sum of all monomials of degree $k$ (resp. $2 k$ ) of $\prod_{i=1}^{n}\left[\left(1+a_{i}\right)\left(1+a_{i}-x\right)\right.$ $\left.\left(1+b_{i}-x\right)^{-1}\right]$ be as $\sum_{s \geq 0} B\left(a_{i}, b_{i}\right) x^{s}$.

(1.1) If $n=p$, then $(r \geqq 2)$

$$
P\left(I_{r}, k, f\right)=\sum_{s, t \geq n-1}(-1)^{s+t} A_{s, t}\left(a_{i}, b_{i}\right) \bar{c}_{s-n+1}(N) f^{*}\left(\bar{c}_{t-n+1}(P)\right)
$$

and

$$
P\left(I_{1}, k, f\right)=\sum_{s \geq n-1}(-1)^{s-n+1} B_{s}\left(a_{i}, b_{i}\right) \bar{c}_{s-n+1}(N) .
$$

(1.2) Let $c_{j}$ mean $c_{j}\left(T N-f^{*}(T P)\right)$ and $\sigma_{s}$, the $s$-th symmetric function of $r-1$ variables $(2,3, \ldots, r) \quad(n=p$ and $r \geqq 2)$. 


$$
P\left(I_{r}, 0, f\right)=\sum_{j=0}^{r-1}\left(\sum_{s=0}^{r-j-1}(-1)^{s} \sigma_{s}\right) c_{j} \bar{c}_{r-j} .
$$

If $P=\mathbb{R}^{n}$ in addition, then

$$
\begin{aligned}
& P\left(I_{r}, 1, f\right)=3 c_{1}\left\{\sum_{s=0}^{r-1}(-1)^{s} \sigma_{s}\left(\sum_{j=0}^{r-s-1}(-1)^{j} c_{j} \bar{c}_{r-j}\right)\right\} \\
& +(1 / 2) r(r-1)\left\{\sum_{s=0}^{r-1}(-1)^{s} \sigma_{s}\left(\sum_{j=0}^{r-s-1}(-1)^{j} c_{j} \bar{c}_{r-j-1}\right)\right\} \\
& +(-1)^{r}(2-r) \quad\left(\sum_{s=0}^{r-1} \sigma_{s} c_{r-s} \bar{c}_{s+1}\right) .
\end{aligned}
$$

These are examples of explicite forms calculated from (1.1).

$(1,3)$ Let $n=p+1$. Let $\sigma_{s}^{\prime}$ be the $s$-th symmetric function of $(3,4, \ldots, r)$ with $\sigma_{s}^{\prime}=0$ for $s \geqq r-1$ and $D(i, j)=\bar{c}_{i} \bar{c}_{j+1}-\bar{c}_{i+1} \bar{c}_{j}(r \geqq 3)$. Then

$$
\begin{aligned}
P( & \left.I_{r}, 0, f\right) \\
= & \sum_{a=0}^{r}(-1)^{a}\left(\sigma_{r-a}^{\prime}+3 \sigma_{r-a-1}^{\prime}+2 \sigma_{r-a-2}^{\prime}\right)\left\{\sum_{s=0}^{a} c_{s}\left(\sum_{u=0}^{a-s} D(u, r-s-u)\right)\right\} \\
& +\sum_{a=0}^{r}(-1)^{a}\left(\sigma_{r-a-1}^{\prime}+2 \sigma_{r-a-2}^{\prime}\right)\left\{\sum_{s=0}^{a} c_{s}\left(\sum_{u=0}^{a-s} D(u+1, r-s-u-1)\right)\right\} .
\end{aligned}
$$

and

$$
P\left(I_{2}, 0, f\right)=2 \bar{c}_{1}\left(\bar{c}_{1}^{2}-\bar{c}_{2}\right) .
$$

Here we must refer to Sergeraert [12] and Gaffney [6]. The first formula of (1.2) has been obtained in [12] (at least in the real case) where any assumption on singularities of $f$ has not been stated. However it turns out to contradict to the result of [6, Theorem 2.2] unless it is provided with some assumption. For instance ours will be one of the most suitable assumptions. The formulas in this section for $r=2$ and $k=0$ have already been given in [11].

\section{§2. Morin Singularities}

Let $J(N, P)$ denote the infinite jet space. In [2] J. M. Boardman has defined so called Boardman submanifolds $\Sigma^{I}(N, P)$ in $J(N, P)$ such that if $j f: N \rightarrow J(N, P)$ is transverse to Boardman submanifolds, then $S^{I_{r}}(f)=(j f)^{-1}\left(\Sigma^{I_{r}}(N, P)\right)$. In this section we review the higher total intrinsic derivatives $d_{t}$ of [2, Definition 7.12]) and some properties of $\Sigma^{I}(N, P)$ for $(i, 0)$ and $I_{r}$. 
Let $\pi_{N}$ and $\pi_{P}$ be the projections of $J(N, P)$ onto $N$ and $P$ mapping a jet onto its source and target respectively. Let $D$ denote the total tangent bundle over $J(N, P)$ isomorphic to $\left(\pi_{N}\right) *(T N)$ （[2, Definition 1.9]). Then we have the homomorphism

$$
d_{1}: D \longrightarrow \pi_{P}^{*}(T P) \quad \text { over } J(N, P) .
$$

We write its restriction over a jet $z$ as $d_{1, z}: D_{z} \rightarrow \pi_{P}^{*}(T P)_{z}$ (Later we use the similar notation). Then $\Sigma^{i}(N, P)$ is defined as the set of all jets $z$ such that $\mathrm{rk}\left(\operatorname{Ker} d_{1, z}\right)$ is $i$. Let $K_{1}$ and $P_{1}$ be the kernel bundle $\operatorname{Ker}\left(d_{1}\right)$ and the cokernel bundle $\operatorname{Cok}\left(d_{1}\right)$ over $\Sigma^{i}(N, P)$ respectively. Next we have the homomorphism

$$
d_{2}: K_{1} \longrightarrow \operatorname{Hom}\left(K_{1}, P_{1}\right) \quad \text { over } \Sigma^{i}(N, P) \text {. }
$$

Define $\Sigma^{i, j}(N, P)$ as the set of all jets $z$ of $\sum^{i}(N, P)$ such that $\operatorname{rk}\left(\operatorname{Ker} d_{2, z}\right)$ is $j$. If $j=1$, we put $K_{2}=\operatorname{Ker}\left(d_{2}\right)$ over $\sum^{i, 1}(N, P)$. Then $\operatorname{Cok}\left(d_{2}\right)$ is isomorphic to $\operatorname{Hom}\left(K_{2}, P_{1}\right)$ over $\Sigma^{i, 1}(N, P)$. The definition of $\Sigma^{I}(N, P)$ for $r \geqq 3$ proceeds by induction on $r$. There exists the homomorphism $(t \geqq 2)$

$$
d_{t+1}: K_{2} \longrightarrow \operatorname{Hom}\left(\stackrel{t}{\otimes} K_{2}, P_{1}\right) \quad \text { over } \sum^{I_{t}}(N, P)
$$

and $\Sigma^{I_{t+1}}(N, P)$ is defined as the set of all jets $z$ of $\Sigma^{I_{t}}(N, P)$ such that $d_{t+1, z}$ is a null homomorphism. We remark that $\Sigma^{\left(I_{t}, 0\right)}(N, P)=$ $\Sigma^{I_{t}}(N, P) \backslash \Sigma^{I_{t+1}}(N, P)$. Furthermore it follows from [2, Lemma 7.13] that $d_{t+1}$ is extended to the homomorphism of the tangent bundle of $\Sigma^{I_{t-1}}(N, P)$ restricted to $\Sigma^{I_{t}}(N, P)$ onto $\operatorname{Hom}\left(K_{1}, P_{1}\right)$ in the case $t=1$ or $\operatorname{Hom}\left(\stackrel{t}{\otimes} K_{2}, P_{1}\right)$ in the case $t \geqq 2$ and that its kernel is the tangent bundle of $\Sigma^{I_{t}}(N, P)$. This yields that the normal bundle of $\Sigma^{i}(N, P)$ in $J(N, P)$ is isomorphic to $\operatorname{Hom}\left(K_{1}, P_{1}\right)$ and one of $\Sigma^{I_{t}}(N, P)$ in $\Sigma^{I_{t-1}}(N, P)$ isomorphic to $\operatorname{Hom}\left(\stackrel{t}{\otimes} K_{2}, P_{1}\right)$ for $t \geqq 2$.

See also the definition of $\Sigma^{i}(N, P)$ and $\Sigma^{i, j}(N, P)$ and the intrinsic derivatives $d_{t}$ in [8], [10] and [11].

\section{§. Lifting of Singularities $S^{I r}(f)$}

For an $n$ dimensional vector space (simply $n$ vector space) $V$, let $G_{i, n-i}(V)$ be the grassmann manifold of all $i$ subspaces of $V$. Let 
$\pi: E \rightarrow M$ be a vector bundle of rank $n$ (simply $n$ vector bundle) over a space $M$ and $E_{m}$, a fibre over a point $m$ of $M$. Let $\pi_{1}: G_{i, n-i}(E)$ $\rightarrow M$ be its associated grassmann bundle whose total space consists of all pairs $(m, a)$ with $m \in M$ and $a \in G_{i, n-i}\left(E_{m}\right)$. Then we have the canonical $i$ vector bundle, $E_{1} \rightarrow G_{i, n-i}(E)$. Any element of $E_{1}$ is written as $(m, a, v)$ for a vector $v$ of $a$. Similarly we can consider $\pi_{2}: G_{1, i-1}\left(E_{1}\right) \rightarrow G_{i, n-i}(E)$ and the line vector bundle, $E_{2} \rightarrow G_{1, i-1}\left(E_{1}\right)$. We write an element of $G_{1, i-1}\left(E_{1}\right)$ as $(m, a, b)$ for $b \in G_{1, i-1}(a)$. For another $p$ vector bundle $\pi^{\prime}: F \rightarrow M$ we consider $G_{n-i, p-n+i}(F)$. Then we write the fibre product of $G_{1, i-1}\left(E_{1}\right)$ and $G_{n-i, p-n+i}(F)$ over $M$ as $G(E, F)$ whose element is written as $(m, a, b, c)$ for $c \in G_{n-i, p-n+i}\left(F_{m}\right)$.

Let $Q=\pi_{P}^{*}(T P)$. By applying these notations for the total tangent bundle $D$ and $Q$ with projections $\pi$ and $\pi^{\prime}$ respectively we obtain vector bundles, $D_{1} \rightarrow G_{i, n-i}(D), D_{2} \rightarrow G_{1, i-1}\left(D_{1}\right)$ and $Q_{1} \rightarrow G_{n-i, p-n+i}(Q)$ and the following commutative diagram with given notations.

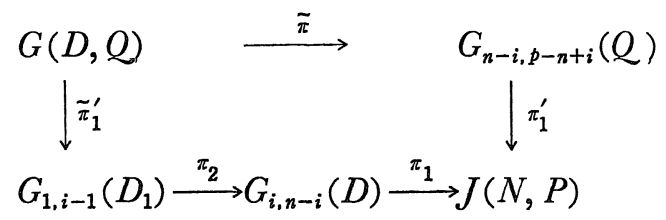

Let $\rho=\pi_{1}^{\prime} \circ \tilde{\pi}\left(=\pi_{1} \circ \pi_{2} \circ \tilde{\pi}_{1}^{\prime}\right)$. We put $\tilde{D}=\rho^{*} D, \tilde{Q}=\rho^{*} Q, \tilde{D}_{1}=\left(\pi_{2} \circ \tilde{\pi}_{1}^{\prime}\right) * D_{1}$, $\tilde{D}_{2}=\left(\tilde{\pi}_{1}^{\prime}\right) * D_{2}$ and $\tilde{Q}_{1}=\tilde{\pi}^{*} Q_{1}$.

We lift the Boardman submanifold $\Sigma^{I_{r}}(N, P)$ of $J(N, P)$ up to that of $G_{i, n-i}(D)$ or $G(D, Q)$ as follows. Let $\tilde{\Sigma}^{i}$ denote the image of the smooth section of $\pi_{1}$ over $\Sigma^{i}(N, P)$ defined by mapping $z \in \Sigma^{i}(N, P)$ onto $\left(z, \operatorname{Ker}\left(d_{1, z}\right)\right) \in G_{i, n-i}(D)$. For $r \geqq 2$ let $\tilde{\Sigma}^{I_{r}}$ denote the image of the smooth section of $\rho$ over $\tilde{\Sigma}^{I_{r}}(N, P)$ defined by mapping $z \in \Sigma^{I_{r}}(N, P)$ onto $\left(z, \operatorname{Ker}\left(d_{1, z}\right), \operatorname{Ker}\left(d_{2, z}\right), \operatorname{Im}\left(d_{1, z}\right)\right)$. Then we have the following

Lemma 3. 1. The normal bundles of $\tilde{\Sigma}^{i}$ in $G_{i, n-i}(D), \tilde{\Sigma}^{I_{2}}$ in $\left(\pi_{2} \circ \tilde{\pi}_{1}^{\prime}\right)^{-1}\left(\tilde{\Sigma}^{i}\right)$ and $\tilde{\Sigma}^{I_{t}}$ in $\tilde{\Sigma}^{I_{t-1}}(t \geqq 3)$ are isomorphic to $\operatorname{Hom}\left(D_{1}, \pi_{1}^{*} Q\right) \mid \tilde{\Sigma^{i}}, \operatorname{Hom}\left(\tilde{D} / \tilde{D}_{1}\right.$, $\left.\tilde{Q} / \tilde{Q}_{1}\right) \oplus \operatorname{Hom}\left(\tilde{D}_{2} \otimes \tilde{D}_{1}, \tilde{Q} / \tilde{Q}_{1}\right) \mid \tilde{\Sigma}^{I_{2}}$ and $\operatorname{Hom}\left(\stackrel{t}{\otimes} \tilde{D}_{2}, \tilde{Q} / \tilde{Q}_{1}\right) \mid \tilde{\Sigma}^{I_{t}}$ respectively.

Proof. We define the following smooth sections as follows. 


$$
\begin{array}{ll}
s_{1}: G_{i, n-i}(D) & \longrightarrow \operatorname{Hom}\left(D_{1}, \pi_{1}^{*} Q\right) \\
s_{2}:\left(\pi_{2} \circ \tilde{\pi}_{1}^{\prime}\right)^{-1}\left(\tilde{\Sigma}^{i}\right) & \longrightarrow \operatorname{Hom}\left(\tilde{D} / \tilde{D}_{1}, \tilde{Q} / \tilde{Q}_{1}\right) \oplus \operatorname{Hom}\left(\tilde{D}_{2}, \operatorname{Hom}\left(\tilde{D}_{1}, \tilde{Q} / \tilde{Q}_{1}\right)\right) \\
\mathrm{s}_{t}: & \tilde{\Sigma}^{I_{t-1}} \longrightarrow \operatorname{Hom}\left(\tilde{D}_{2}, \operatorname{Hom}\left(\tilde{D}_{2}, \tilde{Q} / \tilde{Q}_{1}\right)\right) \quad(t \geqq 3) .
\end{array}
$$

For an element $(z, a) \in G_{i, n-i}(D)$, let $s_{1}(z, a)=d_{1, z} \mid a$. Since $d_{1, z}$ induces a homomorphism $d_{1, z}^{\prime}: D_{z} / \operatorname{Ker}\left(d_{1, z}\right) \rightarrow Q_{z} / \operatorname{Im}\left(d_{1, z}\right)$ we define $s_{2}$ by

$$
s_{2}\left(z, \operatorname{Ker}\left(d_{1, z}\right), b, \operatorname{Im}\left(d_{1, z}\right)\right)=d_{1, z}^{\prime} \oplus d_{2, z} \mid b \text {. }
$$

Let

$$
s_{t}\left(z, \operatorname{Ker}\left(d_{1, z}\right), \operatorname{Ker}\left(d_{2, z}\right), \operatorname{Im}\left(d_{1, z}\right)\right)=d_{t, z} \mid \operatorname{Ker}\left(d_{2, z}\right) .
$$

It follows from the definition of $\tilde{\Sigma}^{I_{t}}$ that the inverse image of the zero section of $s_{t}$ is $\tilde{\Sigma}^{I_{t}}(t \geqq 1)$. Therefore it is enough for the lemma to show that $d_{t}$ is transverse to the zero section. It follows from Porteous [10, Section 1] in the case $t=1$. The case $t=2$ is also proved by the similar arguments in Ronga [11, Proposition 2.1] (Another definition of $d_{2}$ in Levine [6] will be helpful). The case $t \geqq 3$ follows from Boardman [2, Lemma 7.13] since $\tilde{\Sigma}^{I_{t}}$ is diffeomorphic to $\Sigma^{I_{t}}$ by the projection.

Q. E. D.

Let $\Omega(N, P)$ be the subspace of $J(N, P)$ consisting of all jets which is of maximal rank or belongs to $\sum^{i, 0}(N, P) \cup \sum^{i, 1}(N, P)$. Then $\Omega(N, P)$ becomes an open set. A smooth map $f: N \rightarrow P$ has only Morin singularities if and only if $j f$ is a map of $N$ into $\Omega(N, P)$. So we may assume that $f$ is transverse to every Boardman submanifold in the sequel. We write $E$ and $F$ for $T N$ and $f^{*}(T P)$ in the following diagram with given notations.

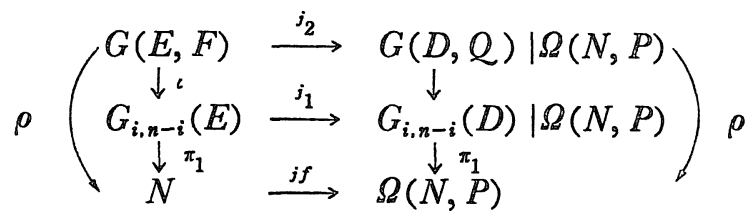

We put $\tilde{S}^{i}(f)=\left(j_{1}\right)^{-1}\left(\tilde{\Sigma^{i}}\right)$ and $\tilde{S}^{I} t(f)=\left(j_{2}\right)^{-1}\left(\tilde{\Sigma}^{I} t\right)(t \geqq 2)$. It follows from the transversality of $j f$ that $\tilde{S}^{I_{t}}(f)$ is mapped onto $S^{I_{t}}(f)$ diffeomorphically by the projection. For simplicity we use the following notation in the sequel $(r \geqq 2)$. 


$$
\begin{gathered}
H_{r}(\tilde{E}, \tilde{F})=\operatorname{Hom}\left(\tilde{E}_{1}, \tilde{F}\right) \oplus \operatorname{Hom}\left(\tilde{E} / \tilde{E}_{1}, \tilde{F} / \tilde{F}_{1}\right) \oplus \operatorname{Hom}\left(\tilde{E}_{2} \otimes \tilde{E}_{1}, \tilde{F} / \tilde{F}_{1}\right) \\
\oplus \operatorname{Hom}\left(\stackrel{r}{t=3}_{t}^{r}\left(\stackrel{t}{\otimes} \tilde{E}_{2}\right), \tilde{F} / \tilde{F}_{1}\right)
\end{gathered}
$$

Theorem 3.2. Let $f$ have only Morin singularities. Then the $k-t h$ Thom polynomial of $S^{I}(f)$ is as follows.

a) $P\left(I_{1}, k, f\right)=\left(\pi_{1}\right) !\left\{W^{k}\left(\pi_{1}^{*} E \oplus \operatorname{Hom}\left(E_{1}, \pi_{1}^{*} E / E_{1}\right)\right.\right.$

$$
\left.\left.-\operatorname{Hom}\left(E_{1}, \pi_{1}^{*} F\right)\right) \chi \operatorname{Hom}\left(E_{1}, \pi_{1}^{*} F\right)\right\}
$$

b) $P\left(I_{r}, k, f\right)=\rho !\left\{W^{k}\left(\tilde{E} \oplus \operatorname{Hom}\left(\tilde{E}_{1}, \tilde{E} / \tilde{E}_{1}\right) \oplus \operatorname{Hom}\left(\tilde{F}_{1}, \tilde{F} / \tilde{F}_{1}\right)\right.\right.$

$$
\left.\left.-H_{r}(\tilde{E}, \tilde{F})\right) \chi H_{r}(\tilde{E}, \tilde{F})\right\} \quad(r \geqq 2) .
$$

where $E=T N$ and $F=f^{*}(T P)$.

Proof. We give a proof of (b) ((a) is proved similarly). We may assume that $j f$ is transverse to every Boardman submanifold. Then it follows from Lemma 3.1 that the normal bundles of $\tilde{S}^{i}(f)$ in $G_{i, n-i}(E), \tilde{S}^{I_{2}}(f)$ in $(\iota)^{-1}\left(\tilde{S}^{i}(f)\right)$ and $\tilde{S}^{I_{t}}(f)$ in $\tilde{S}^{I_{t-1}}(f)(t \geqq 3)$ are $\operatorname{Hom}\left(E_{1}, \pi_{1}^{*} F\right)\left|\tilde{S}^{i}(f), \operatorname{Hom}\left(\tilde{E} / \tilde{E}_{1}, \tilde{F} / \tilde{F}_{1}\right) \oplus \operatorname{Hom}\left(\tilde{E}_{2} \otimes \tilde{E}_{1}, \tilde{F} / \tilde{F}_{1}\right)\right|$ $\tilde{S}^{I_{2}}(f)$ and $\operatorname{Hom}\left(\otimes \tilde{E}_{2}, \tilde{F} / \tilde{F}_{1}\right) \mid \tilde{S}^{I_{t}}(f)$ respectively. By this fact together with the proof of Lemma 3.1 their dual classes are given by the Euler classes (denoted by $\chi$ ) of the corresponding normal bundles coming from the cohomology group of $G(E, F)$ (cf. [11]). That is, the dual class of the fundamental class of $\tilde{S}^{I_{r}}(f)$ is given by $\chi\left(H_{r}(\tilde{E}, \tilde{F})\right)$. The Poincaré dual of the $(n-d-k)$-th Stiefel Whitney homology class $\tilde{s}_{n-d-k}$ of $\tilde{S}^{I_{r}}(f)$ is $W^{k}\left(\tilde{S}^{I_{r}}(f)\right)$ and the tangent bundle of $\tilde{S}^{I_{r}}(f)$ is given by $\left(T\left(G(E, F) / H_{r}(\tilde{E}, \tilde{F})\right) \mid \tilde{S}^{I_{r}}(f)\right.$. Hence the Poincaré dual of $j^{*}\left(\tilde{s}_{n-d-k}\right)$ is equal to

$$
W^{k}\left(T\left(G(E, F) / H_{r}(\tilde{E}, \tilde{F})\right) \chi\left(H_{r}(\tilde{E}, \tilde{F})\right)\right.
$$

where $j: \tilde{S}^{I_{r}}(f) \rightarrow G(E, F)$ is the inclusion. It is easy to see that $T(G(E, F))$ is given by $\tilde{E} \oplus \operatorname{Hom}\left(\tilde{E}_{1}, \tilde{E} / \tilde{E}_{1}\right) \oplus \operatorname{Hom}\left(\tilde{F}_{1}, \tilde{F} / \tilde{F}_{1}\right)$. Since $\tilde{S}^{I_{r}}(f)$ is diffeomorphic to $S^{I_{r}}(f)$ by the projection, $\tilde{s}_{n-d-k}$ is mapped onto $s_{n-d-k}$ of Section 1. Therefore Theorem 3.2 follows from the definition of the Gysin homomorphism.

Q.E. D. 


\section{§. Calculation of Thom Polynomials}

In the rest of the paper $c=1+c_{1}+c_{2}+\ldots$ denotes the Stiefel Whitney class in the real case or the Chern class in the complex case. The coefficient group of (co) homology group is $\mathbb{Z} / 2 \mathbb{Z}$ (resp. $\mathbb{Z}$ ) in the real (resp. complex) case. For an $n$-vector bundle $\pi: E \rightarrow M$, we put $X=c_{1}(E)$ for the line bundle $E_{1} \rightarrow G_{1, n-1}(E)$. Then we have the following well known proposition (see, for example, [10, Proposition $0.3]$ and [13].

Proposition 4.1. a) The kernel of the $H^{*}(M)$-homomorphism $\phi: H^{*}(M)[x] \rightarrow H^{*}\left(G_{1, n-1}(E)\right)$ defined by $\phi\left(a x^{t}\right)=\pi_{1}^{*}(a) X^{t}$ is the ideal generated by

$$
\sum_{j=0}^{n}(-1)^{n-j} c_{j}(E) x^{n-j} .
$$

b) $\left(\pi_{1}\right) !\left(X^{n+l}\right)=(-1)^{l+1} \bar{c}_{l+1}(E)$ 。

In this section we assume $n=p$. Then we have $G_{1, n-1}(E)=G_{1, i-1}\left(E_{1}\right)$ and $E_{1}=E_{2}$. We represent the characteristic classes by using given variables of degree 1 (resp. 2) as follows.

$$
\begin{array}{ll}
c(E)=\prod_{i=1}^{n}\left(1+a_{i}\right), & c(F)=\prod_{i=1}^{n}\left(1+b_{i}\right) \\
c\left(\rho^{*} E\right)=\prod_{i=1}^{n}\left(1+\tilde{a}_{i}\right), & c\left(\rho^{*} F\right)=\prod_{i=1}^{n}\left(1+\tilde{b}_{i}\right) \\
c\left(\tilde{E}_{1}\right)=1+x, & c\left(\tilde{F} / \tilde{F}_{1}\right)=1+y
\end{array}
$$

Since $\operatorname{Hom}\left(\tilde{E}_{1}, \tilde{E}_{1}\right)$ and $\operatorname{Hom}\left(\tilde{F} / \tilde{F}_{1}, \tilde{F} / \tilde{F}_{1}\right)$ are trivial line bundles, we have

$$
\begin{aligned}
& c\left(\operatorname{Hom}\left(\tilde{E}_{1}, \tilde{E} / \tilde{E}_{1}\right)\right)=c\left(\operatorname{Hom}\left(\tilde{E}_{1}, \tilde{E}\right)\right)=\prod_{i=1}^{n}\left(1-x+\tilde{a}_{i}\right) \\
& c\left(\operatorname{Hom}\left(\tilde{F}_{1}, \tilde{F} / \tilde{F}_{1}\right)\right)=c\left(\operatorname{Hom}\left(\tilde{F}, \tilde{F} / \tilde{F}_{1}\right)\right)=\prod_{i=1}^{n}\left(1-\tilde{b}_{i}+y\right) \\
& c\left(\operatorname{Hom}\left(\tilde{E}_{1}, \tilde{F}\right)\right)=\prod_{i=1}^{n}\left(1-x+\tilde{b}_{i}\right) \\
& c\left(\operatorname{Hom}\left(\stackrel{t}{\otimes} \tilde{E}_{2}, \tilde{F} / \tilde{F}_{1}\right)\right)=c\left(\operatorname{Hom}\left(\stackrel{t}{\otimes}^{t} \tilde{E}_{1}, \tilde{F} / \tilde{F}_{1}\right)\right)=1-t x+y \\
& c\left(\operatorname{Hom}\left(\tilde{E} / \tilde{E}_{1}, \tilde{F} / \tilde{F}_{1}\right)\right)=c\left(\operatorname{Hom}\left(\tilde{E}, \tilde{F} / \tilde{F}_{1}\right)\right) c\left(\operatorname{Hom}\left(\tilde{E}_{1}, \tilde{F} / \tilde{F}_{1}\right)\right)^{-1}
\end{aligned}
$$

From these formulas we have that 


$$
c_{k}\left(\tilde{E} \oplus \operatorname{Hom}\left(\tilde{E_{1}}, \tilde{E} / \tilde{E}_{1}\right) \oplus \operatorname{Hom}\left(\tilde{F}_{1}, \tilde{F} / \tilde{F}_{1}\right)-H_{r}(\tilde{E}, \tilde{F})\right)
$$

is the sum of all monomials of degree $k$ (resp. $2 k$ ) of the following polynomial.

$$
\begin{aligned}
\prod_{i=1}^{n}\left[\left(1+\tilde{a}_{i}\right)\right. & \left.\left(1+\tilde{a}_{i}-x\right)\left(1-\tilde{b}_{i}+y\right)\left(1+\tilde{b}_{i}-x\right)^{-1}\left(1-\tilde{a}_{i}+y\right)^{-1}\right] \\
& \times(1-x+y) \prod_{t=2}^{r}(1-t x+y)^{-1}
\end{aligned}
$$

Furthermore we have

$$
\begin{aligned}
\chi\left(H_{r}(\tilde{E}, \tilde{F})\right) & =\prod_{i=1}^{n}\left(\tilde{b}_{i}-x\right)\left\{\sum_{l=0}^{n-1}\left(\sum_{j=0}^{n-l-1}(-1)^{j} c_{j}(\tilde{E}) x^{n-l-j-1}\right) y^{l}\right\} \\
& \times \prod_{t=2}^{r}(-t x+y)
\end{aligned}
$$

Now we prove the formulas of Section 1 by beginning with $(1,1)$. We define $A_{s, t}\left(\tilde{a}_{i}, \tilde{b}_{i}\right)$ similarly as $A_{s, t}\left(a_{i}, b_{i}\right)$ in Section 1 . Then it follows from Theorem 3.2 that

$$
P\left(I_{r}, k, f\right)=\rho !\left(\sum_{s, t \geq 0} A_{s, t}\left(\tilde{a}_{i}, \tilde{b}_{i}\right) x^{s} y^{t}\right) .
$$

Hence Proposition 4.1 yields the following.

$$
\begin{aligned}
P\left(I_{r}, k, f\right) & =\sum_{s, t \geq 0} A_{s, t}\left(a_{i}, b_{i}\right)(\rho !)\left(x^{s} y^{t}\right) \\
& =\sum_{s, t \geq n-1}(-1)^{s+t} A_{s, t}\left(a_{i}, b_{i}\right) \bar{c}_{s-n+1}(N) f^{*} \bar{c}_{t-n+1}(P)
\end{aligned}
$$

The calculation of $P\left(I_{1}, k, f\right)$ is similar.

Next we prove (1.2) and (1.3). Let $i: P \rightarrow R^{p+m}$ be an embedding and $\nu$, an open disk normal bundle $U(P)$ of $P$ in $\boldsymbol{R}^{p+m}$. For $f: N \rightarrow P$ we consider the induced bundle $f^{*} U(P)$ with the diagram

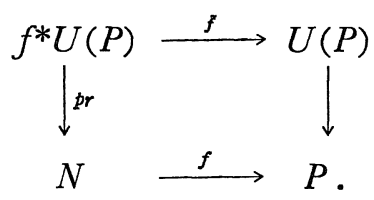

It follows from the geometrical interpretation of Morin singularities that $S^{I_{t}}(\tilde{f})=(p r)^{-1}\left(S^{I_{t}}(f)\right)$. Therefore we have $(p r)^{*}\left(P\left(I_{r}, 0, f\right)\right)$ $= \pm P\left(I_{r}, 0, \tilde{f}\right)$. This means $i^{*}\left(P\left(I_{r}, 0, \tilde{f}\right)\right)= \pm P\left(I_{r}, 0, f\right)$ for the inclusion $i: N \rightarrow f^{*} U(P)$. So we may suppose that $E=T N \oplus f^{*} \nu$ and $F=f^{*}(T P \oplus \nu)\left(=f^{*}\left(T R^{p+m}\right)\right)$ in the calculation. Since $F$ is a trivial bundle, we have $\rho !\left(y^{j}\right)=0$ for $j \neq n+m-1$. By replacing $n, b_{i}$ and $c_{j}(E)$ by $n+m, 0$ and $c_{j}\left(T N-f^{*} T P\right.$ ) (simply denoted by $c_{j}$ ), we have 


$$
\begin{aligned}
P\left(I_{r}, 0, f\right) & =(\rho !)\left\{(-x)^{n+m} \sum_{l=0}^{n+m-1}\left(\sum_{j=0}^{n+m-l-1}(-1)^{j} c_{j} x^{n+m-l-j-1}\right) y^{l}\right. \\
& \left.\left.\times \sum_{s=0}^{r-1}(-1)^{s} \sigma_{s} x^{s} y^{r-1-s}\right)\right\} \\
= & (-1)^{n+m}(\rho !)\left\{\sum_{s=0}^{r-1}(-1)^{s} \sigma_{s}\left(\sum_{j=0}^{r-s-1}(-1)^{j} c_{j} x^{n+m+r-j-1}\right) y^{n+m-1}\right\} \\
= & (-1)^{n+m+r} \sum_{s=0}^{r-1}(-1)^{s} \sigma_{s}\left(\sum_{j=0}^{r-s-1} c_{j} \bar{c}_{r-j}\right) \\
= & (-1)^{n+m+r} \sum_{j=0}^{r-1}\left(\sum_{s=0}^{r-j-1}(-1)^{s} \sigma_{s}\right) c_{j} \bar{c}_{r-j} .
\end{aligned}
$$

The second formula of (1.2) will be shown similarly.

For the calculation of $P\left(I_{r}, k, f\right)$ for $n>p$, the argument in a proof of (1.2) and [4, Corollary 2] will be helpful. Let $E_{2}^{\prime}$ be the canonical line bundle over $G_{1, n+m-1}(E)$ with projection $\rho_{1}: G_{1, n+m-1}(E)$ $\rightarrow N$. Then we canonically identify $G_{1,1}\left(E_{1}\right)$ with $G_{1, n+m-2}\left(\rho_{1}^{*} E / E_{2}^{\prime}\right)$ and with the Flag bundle $F_{2,1}(E)$ consisting of all pairs of 2 subspaces and their 1 subspaces in every fibre of $E$. We use the given notation of the following commutative diagram.

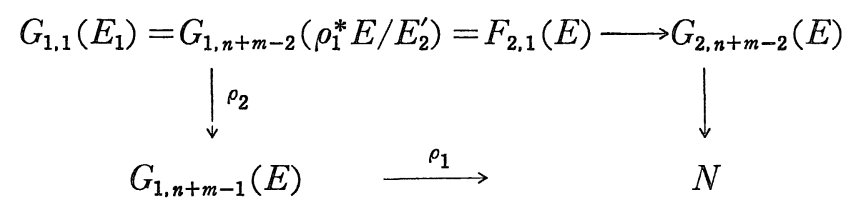

Then we can compute $\rho !\left(\chi\left(H_{r}\right)\right)$ by passing through $\left(\rho_{1} \circ \rho_{2}\right)$ !. In the calculation every induced bundle $h^{*} L$ of a bundle $L$ by a map $h$ will be denoted by the same letter $L$ for simplicity. The coefficient of $y^{n+m-1}$ of $\chi\left(H_{r}\right)$ is given as follows.

$$
\begin{aligned}
&(*) c_{2}\left(E_{1}^{*}\right)^{n+m}\left\{\sum_{a=2}^{n+m-1}(-1)^{r} c_{a}\left(E / E_{1}\right) \sigma_{r-a}\left(c_{1}\left(E_{2}\right)\right)^{r-a}\right. \\
&+\sum_{a=1}^{n+m-1}(-1)^{r} c_{a}\left(E / E_{1}\right) \sigma_{r-a-1}\left(c_{1}\left(E_{2}\right)\right)^{r-a-1}\left(2 c_{1}\left(E_{2}\right)+c_{1}\left(E_{1}\right)\right) \\
&+\sum_{a=0}^{n+m-1}(-1)^{r} c_{a}\left(E / E_{1}\right) \sigma_{r-a-2}\left(c_{1}\left(E_{2}\right)\right)^{r-a}\left(c_{1}\left(E_{2}\right)^{2}+c_{1}\left(E_{1}\right) c_{1}\left(E_{2}\right)\right. \\
&\left.\left.+c_{2}\left(E_{1}\right)\right)\right\}
\end{aligned}
$$

Then it follows from Proposition 4. 1 that

$$
\begin{aligned}
P((2,1, \ldots, 1), 0, f) & =\rho !\left(\chi\left(H_{r}\right)\right) \\
& =\left(\rho_{1} \circ \rho_{2}\right) !\left(\text { the coefficient of } y^{n+m-1} \text { of } \chi\left(H_{r}\right)\right) .
\end{aligned}
$$

This is calculated as follows. 


$$
\begin{aligned}
& \left(\rho_{1} \circ \rho_{2}\right) !\left\{c_{2}\left(E_{1}^{*}\right)^{n+m-1}\left(\sum_{a=2}^{n+m-1}(-1)^{r} c_{a}\left(E / E_{1}\right) \sigma_{r-a}\left(c_{1}\left(E_{2}\right)\right)^{r-a}\right)\right\} \\
& =(-1)^{r}\left(\rho_{1} \circ \rho_{2}\right) !\left\{c _ { 1 } ( E _ { 1 } / E _ { 2 } ) ^ { n + m - 1 } c _ { 1 } ( E _ { 2 } ) ^ { n + m - 1 } \left\{\sum_{a=2}^{n+m-1} \sigma_{r-a} c_{1}\left(E_{2}\right)^{r-a}\right.\right. \\
& \left.\left.\times\left(\sum_{s=0}^{a} c_{s}(E) \bar{c}_{a-s}\left(E_{1}\right)\right)\right\}\right\} \\
& =(-1)^{r}\left(\rho_{1} \circ \rho_{2}\right) !\left\{c _ { 1 } ( E _ { 1 } / E _ { 2 } ) ^ { n + m - 1 } c _ { 1 } ( E _ { 2 } ) ^ { n + m - 1 } \left\{\sum_{a=2}^{n+m-1} \sigma_{r-a} c_{1}\left(E_{2}\right)^{r-a}\right.\right. \\
& \left.\left.\times\left[\sum_{s=0}^{a}(-1)^{a-s} c_{s}(E)\left(\sum_{u=0}^{a-s} c_{1}\left(E_{1} / E_{2}\right)^{u} c_{1}\left(E_{2}\right)^{a-s-u}\right)\right]\right\}\right\} \\
& =(-1)^{r}\left(\rho_{1}\right) !\left\{\sum _ { a = 2 } ^ { n + m - 1 } \sigma _ { r - a } \left\{\sum _ { s = 0 } ^ { a } ( - 1 ) ^ { a - s } c _ { s } ( E ) \left(\sum_{u=0}^{a-s}(-1)^{u+1}\right.\right.\right. \\
& \left.\left.\left.\times \bar{c}_{u+1}\left(E / E_{2}^{\prime}\right) c_{1}\left(E_{2}^{\prime}\right)^{n+m+r-s-u}\right)\right\}\right\} \\
& =\sum_{a=2}^{n+m-1}(-1)^{a} \sigma_{r-a}\left\{\sum_{s=0}^{a} c_{s}(E)\left(\sum_{u=0}^{a-s} D(u, r-s-u)\right)\right\} \\
& =\sum_{a=0}^{r}(-1)^{a} \sigma_{r-a}\left\{\sum_{s=0}^{a} c_{s}(E)\left(\sum_{u=0}^{a-s} D(u, r-s-u)\right)\right\}
\end{aligned}
$$

By the similar calculation it is shown that the second and the third terms of $\left(^{*}\right)$ are mapped onto

$$
\begin{aligned}
2\left\{\sum _ { a = 0 } ^ { r } ( - 1 ) ^ { a } \sigma _ { r - a - 1 } \left\{\sum_{s=0}^{a} c_{s}(E)\right.\right. & \left(\sum_{u=0}^{a-s}(2 D(u, r-s-u)\right. \\
& +D(u+1, r-s-u-1)))\}\}
\end{aligned}
$$

and

$$
\begin{aligned}
\sum_{a=0}^{r}(-1)^{a} \sigma_{r-a-2}\left\{\sum _ { s = 0 } ^ { a } c _ { s } ( E ) \left(_{u=0}^{a-s}\right.\right. & (2 D(u, r-s-u) \\
& +2 D(u+1, r-s-u-1)))\}
\end{aligned}
$$

respectively. Since $E=T N \oplus f^{*}(\nu)$, we have $c_{j}(E)=c_{j}\left(T N-f^{*}(T P)\right)$.

\section{References}

[1] Ando, Y., On the elimination of Morin singularities, J. Math. Soc. Japan, 37 (1985), 471-488.

[2] Boardman, J. M., Singularities of differenciable maps, IHES. Publ. Math., 33 (1967), 21-57.

[3] Damon, J., Thom polynomials for contact class singularities, Thesis, Harvard Univ., (1972).

[4] The Gysin homomorphisms for flag bundles; applications, Amer. J. Math., 96 (1974), 248-260.

[5] Fukuda, T., Topology of folds, cusps and Morin singularities, Preprint. 
[6] Gaffney, T., The Thom polynomials of $\overline{\Gamma^{1111}}$, Proc. Symp. Pure Math., A. M. S., 40 (1983), 399-408.

[7] Haefliger, A. and Kosinski, A., Un théorèm de Thom sur les singularités des applications différentiable, Séminaire H. cartan E. N. S., 8 (1956/57).

[8] Levin, H. I., Singularities of differentiable mappings, Proc. Liverpool Singularities, Springer Lecture Notes, 192 (1971), 1-89.

[9] Morin, B., Formes canonique des singularités d'une application différentiables, $C . R$. Acad. Sc. Paris, 260 (1965), 5662-5665, 6503-6506.

[10] Porteous, I. R., Simple singularities, Proc. Liverpool Singularities, Springer Lecture Notes, 192 (1971), 286-307.

[11] Ronga, F., Le calcule des classes duales singularités de Boardman d'ordre deux, Comment. Math. Helv., 47 (1972), 15-35.

[12] Sergeraert, F., Expression explicite de certains polynômes de Thom, C. R. Acad。 Sc. Paris, 276 (1973), 1661-1663.

[13] Stong, R., Notes on Cobordism Theory, Princeton Math. Notes, Princeton Univ. Press, 1968.

[14] Thom, R., Les singularités des applications différentiables, Ann. Inst. Fourier, 6 (195556), 43-87. 
